Acta Crystallographica Section E

\section{Structure Reports}

Online

ISSN 1600-5368

\title{
A 1:1 molecular complex of dicyclohexylamine and cyclohexanone oxime
}

The molecules of the title complex, $\mathrm{C}_{12} \mathrm{H}_{23} \mathrm{~N} \cdot \mathrm{C}_{6} \mathrm{H}_{11} \mathrm{~N}$, are linked together in chains by $\mathrm{O}-\mathrm{H} \cdots \mathrm{N}$ and $\mathrm{N}-\mathrm{H} \cdots \mathrm{O}$ hydrogen bonds.

\section{Comment}

Both components of the title complex, (I) (Fig. 1), show the expected molecular geometries and both cyclohexane rings of the dicyclohexylamine molecule adopt the chair conformation. The conformation of the oxime six-membered ring is halfchair, very similar to that observed in the structure of cyclohexanone oxime itself (Olivato et al., 2001). The geometrical parameters of the oxime fragment show standard values for oximes (Chertanova et al.,1994). The most interesting feature of this structure is the system of hydrogen bonds. The oxime hydrogen bonds were first classified by Bertolasi et al. (1982) and divided into three groups. The structures where the oxime unit is a donor group and forms one hydrogen bond are in group $A$. The structures with an additional hydrogen bond (oxime $\mathrm{N}$ atom is a hydrogen-bond acceptor) form group $B$ and the structures with one more hydrogen bond, where the oxime $\mathrm{O}$ atom is the acceptor, are regarded as group $C$. The later and more elaborate classification introduced by Chertanova et al. (1994) shows very few examples of other motifs. In both cases the analyses did not include the directionality of the hydrogen bonds and supposed them to be in the plane of oxime group. In the case of complex (I), one of the two hydrogen bonds is the classical type $A \mathrm{O}-\mathrm{H} \cdots \mathrm{N}$ bond (Table 1 and Fig. 2). However, the geometry of the second hydrogen bond cannot be regarded as a pure $B$ - or $C$-type bond. The $\mathrm{N}-\mathrm{H}$ vector is not in the plane of the oxime group and is pointed toward the centre of the oxime $\mathrm{O}-\mathrm{N}$ bond of an adjacent molecule. Therefore, this can be classified as a weak bifurcated hydrogen bond and the overall resulting bonding of the oxime unit in complex (I) is intermediate between $B$ - and $C$-types.

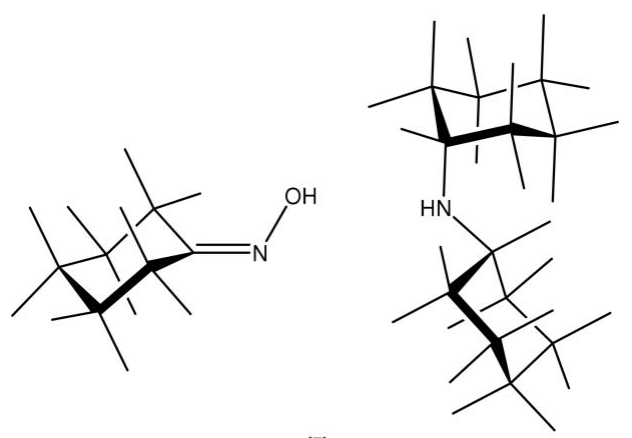

(I)
Received 24 March 2006 Accepted 19 April 2006 


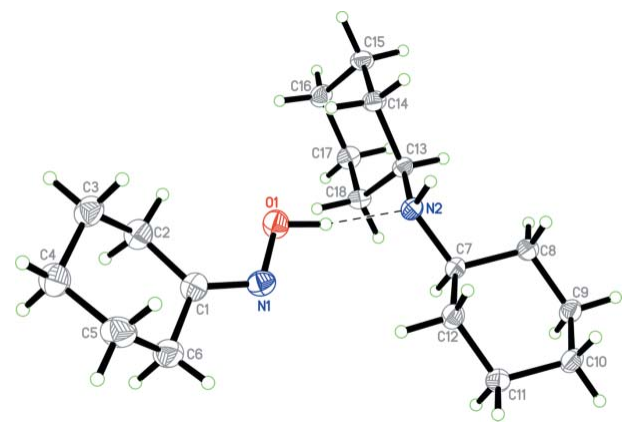

Figure 1

Structure of the molecular complex (I). Displacement ellipsoids are shown at the $50 \%$ probability level. The dashed line indicates a hydrogen bond.

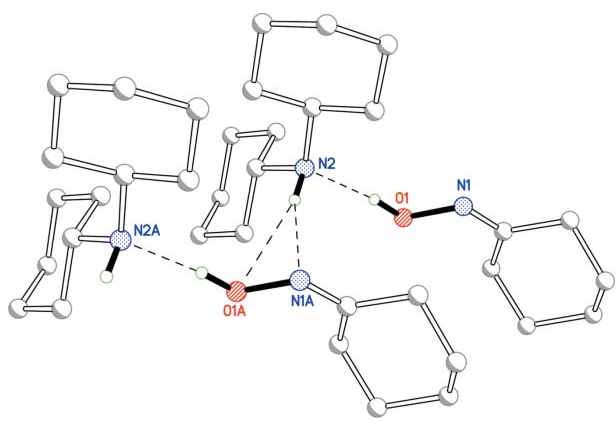

Figure 2

Hydrogen bonds (dashed lines) in the structure of (I). $\mathrm{H}$ atoms not involved in the interactions shown have been omitted.

The hydrogen bonds link the molecules into chains, parallel to the $c$ axis. The oxime molecules in the chains are also connected by weak $\mathrm{C} 1-\mathrm{H} 21 \cdots \mathrm{O} 1(\mathrm{H} \cdots \mathrm{O}=2.71 \AA)$ interactions. The chains form loose layers perpendicular to the $a$ direction (Fig. 3)

\section{Experimental}

A solution of an organic compound $(10 \mathrm{mg})$ in dicyclohexylamine $(0.3 \mathrm{ml})$ was heated at $423 \mathrm{~K}$ in an open vessel for 2-3 min. The molecular complex (I) crystallized serendipitously as colourless needles after cooling of the solution, followed by standing at room temperature for several months. Presumably, the compound is a product of some oxidative conversion of the solvent.

\section{Crystal data}

$\mathrm{C}_{12} \mathrm{H}_{23} \mathrm{~N} \cdot \mathrm{C}_{6} \mathrm{H}_{11} \mathrm{NO}$

$M_{r}=294.47$

Orthorhombic, Pna2 1

$a=29.599(6) \AA$

$b=11.359(2) \AA$

$c=5.1586(10) \AA$

$V=1734.3(6) \AA^{3}$

\section{Data collection}

Bruker SMART CCD 6000
diffractometer

$\omega$ scans

Absorption correction: multi-scan

(SADABS; Bruker, 2003)

$T_{\min }=0.953, T_{\max }=0.998$

$$
\begin{aligned}
& Z=4 \\
& D_{x}=1.128 \mathrm{Mg} \mathrm{m}^{-3} \\
& \text { Mo- } K \alpha \text { radiation } \\
& \mu=0.07 \mathrm{~mm}^{-1} \\
& T=120(2) \mathrm{K} \\
& \text { Needle, colourless } \\
& 0.70 \times 0.04 \times 0.03 \mathrm{~mm}
\end{aligned}
$$

12334 measured reflections 2118 independent reflections 1714 reflections with $I>2 \sigma(I)$ $R_{\text {int }}=0.059$

$\theta_{\max }=27.0^{\circ}$
Figure 3

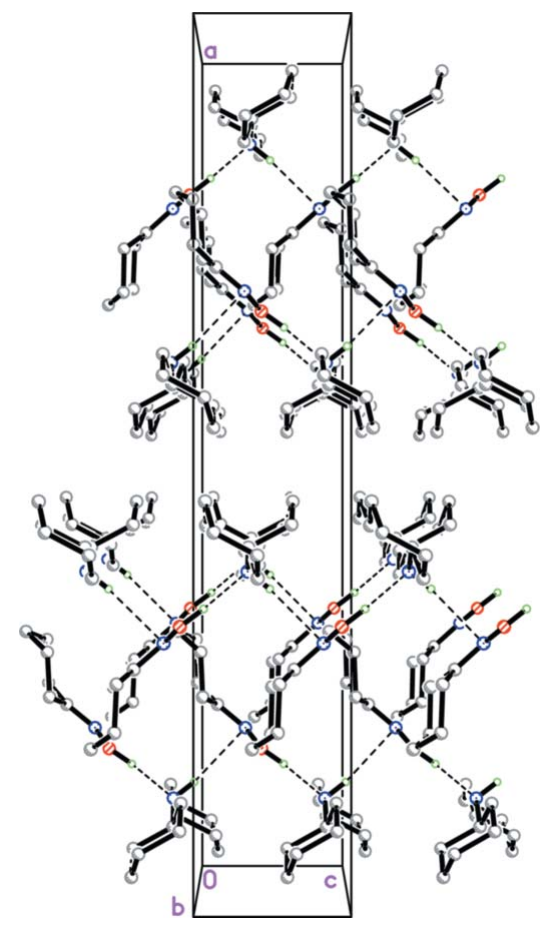

Packing of the molecules in the structure of (I), viewed along the $b$ axis. $\mathrm{H}$ atoms not involved in the hydrogen bonds (dashed lines) shown have been omitted.

Refinement

Refinement on $F^{2}$

$R\left[F^{2}>2 \sigma\left(F^{2}\right)\right]=0.037$

$w R\left(F^{2}\right)=0.094$

$S=1.05$

2118 reflections

326 parameters

All $\mathrm{H}$-atom parameters refined

$$
\begin{gathered}
w=1 /\left[\sigma^{2}\left(F_{\mathrm{o}}{ }^{2}\right)+(0.03 P)^{2}\right. \\
+0.6 P] \\
\text { where } P=\left(F_{\mathrm{o}}{ }^{2}+2 F_{\mathrm{c}}{ }^{2}\right) / 3 \\
(\Delta / \sigma)_{\max }<0.001 \\
\Delta \rho_{\max }=0.19 \mathrm{e}^{-3} \\
\Delta \rho_{\min }=-0.18 \mathrm{e}^{-3}
\end{gathered}
$$

\section{Table 1}

Selected geometric parameters $\left(\AA{ }^{\circ}{ }^{\circ}\right)$.

\begin{tabular}{llll}
\hline $\mathrm{O} 1-\mathrm{N} 1$ & $1.419(3)$ & $\mathrm{N} 2-\mathrm{C} 13$ & $1.477(3)$ \\
$\mathrm{N} 1-\mathrm{C} 1$ & $1.292(3)$ & $\mathrm{N} 2-\mathrm{C} 7$ & $1.484(3)$ \\
& & & \\
$\mathrm{C} 1-\mathrm{N} 1-\mathrm{O} 1$ & $111.7(2)$ & $\mathrm{N} 2-\mathrm{C} 7-\mathrm{C} 8$ & $113.1(2)$ \\
$\mathrm{C} 13-\mathrm{N} 2-\mathrm{C} 7$ & $115.91(16)$ & $\mathrm{C} 12-\mathrm{C} 7-\mathrm{C} 8$ & $110.0(2)$ \\
$\mathrm{N} 1-\mathrm{C} 1-\mathrm{C} 6$ & $116.7(2)$ & $\mathrm{N} 2-\mathrm{C} 13-\mathrm{C} 18$ & $111.0(2)$ \\
$\mathrm{N} 1-\mathrm{C} 1-\mathrm{C} 2$ & $127.0(2)$ & $\mathrm{N} 2-\mathrm{C} 13-\mathrm{C} 14$ & $108.67(18)$ \\
$\mathrm{C} 6-\mathrm{C} 1-\mathrm{C} 2$ & $116.3(2)$ & $\mathrm{C} 18-\mathrm{C} 13-\mathrm{C} 14$ & $109.7(2)$ \\
$\mathrm{N} 2-\mathrm{C} 7-\mathrm{C} 12$ & $109.04(18)$ & & \\
\hline
\end{tabular}

Table 2

Hydrogen-bond geometry $\left(\AA,^{\circ}\right)$.

\begin{tabular}{lllll}
\hline$D-\mathrm{H} \cdots A$ & $D-\mathrm{H}$ & $\mathrm{H} \cdots A$ & $D \cdots A$ & $D-\mathrm{H} \cdots A$ \\
\hline $\mathrm{O} 1-\mathrm{H} 1 \mathrm{O} \cdots \mathrm{N} 2$ & $0.98(5)$ & $1.85(5)$ & $2.818(3)$ & $167(4)$ \\
$\mathrm{N} 2-\mathrm{H} 2 \mathrm{~N} \cdots \mathrm{O} 1^{\mathrm{i}}$ & $0.90(3)$ & $2.62(3)$ & $3.509(3)$ & $167(2)$ \\
$\mathrm{N} 2-\mathrm{H} 2 \mathrm{~N} \cdots \mathrm{N} 1^{\mathrm{i}}$ & $0.90(3)$ & $2.59(3)$ & $3.451(3)$ & $160(2)$ \\
$\mathrm{C} 2-\mathrm{H} 21 \cdots \mathrm{O} 1^{\mathrm{ii}}$ & $1.04(3)$ & $2.71(3)$ & $3.686(3)$ & $155(2)$ \\
\hline
\end{tabular}

Symmetry codes: (i) $x, y, z+1$; (ii) $x, y, z-1$.

All $\mathrm{H}$ atoms were located in a difference Fourier map and refined isotropically [C-H = 0.94 (3)-1.05 (4) $\mathrm{A}]$. 
Data collection: SMART (Bruker, 2003); cell refinement: SAINT (Bruker, 2003); data reduction: $S A I N T$; $\operatorname{program}(\mathrm{s})$ used to solve structure: SHELXTL (Bruker, 2003); program(s) used to refine structure: SHELXTL; molecular graphics: SHELXTL; software used to prepare material for publication: $S H E L X T L$.

The manuscript was prepared with the beta test version 1.0.0. of the program publCIF to be released by the IUCr and with the program modiCIFer to be released by the University of Wisconsin-Madison.

\section{References}

Bertolasi, V., Gilli, G. \& Veronese, A. C. (1982). Acta Cryst. B38, 502511.

Bruker (2003). SADABS (Version 2.05), SAINT (Version 6.45), SHELXTL (Version 6.14) and SMART (Version 5.622). Bruker AXS Inc., Madison, Wisconsin, USA.

Chertanova, L., Pascard, C. \& Sheremetev, A. (1994). Acta Cryst. B50, 708716.

Olivato, P. R., Ribeiro, D. S., Zukerman-Schpector, J. \& Bombieri, G. (2001). Acta Cryst. B57, 705-713. 


\title{
supporting information
}

Acta Cryst. (2006). E62, o2053-o2055 [https://doi.org/10.1107/S1600536806014310]

\section{A 1:1 molecular complex of dicyclohexylamine and cyclohexanone oxime}

\author{
Olga V. Chetina, Dmitry S. Yufit and Judith A. K. Howard
}

dicyclohexylamine-cyclohexanone oxime (1/1)

Crystal data

$\mathrm{C}_{12} \mathrm{H}_{23} \mathrm{~N} \cdot \mathrm{C}_{6} \mathrm{H}_{11} \mathrm{NO}$

$M_{r}=294.47$

Orthorhombic, Pna2 $_{1}$

Hall symbol: $\mathrm{P} 2 \mathrm{c}-2 \mathrm{n}$

$a=29.599(6) \AA$

$b=11.359(2) \AA$

$c=5.1586(10) \AA$

$V=1734.3(6) \AA^{3}$

$Z=4$

$F(000)=656$

\section{Data collection}

\section{Bruker SMART CCD 6000}

diffractometer

Radiation source: fine-focus sealed tube

Graphite monochromator

$0.30^{\circ} \omega$ scans

Absorption correction: multi-scan

(SADABS; Bruker, 2003)

$T_{\min }=0.953, T_{\max }=0.998$

Refinement

Refinement on $F^{2}$

Least-squares matrix: full

$R\left[F^{2}>2 \sigma\left(F^{2}\right)\right]=0.037$

$w R\left(F^{2}\right)=0.094$

$S=1.05$

2118 reflections

326 parameters

1 restraint

Primary atom site location: structure-invariant direct methods
$D_{\mathrm{x}}=1.128 \mathrm{Mg} \mathrm{m}^{-3}$

Melting point: $58^{\circ} \mathrm{C}$ decomp. $\mathrm{K}$

Mo $K \alpha$ radiation, $\lambda=0.71073 \AA$

Cell parameters from 2345 reflections

$\theta=2.3-25.1^{\circ}$

$\mu=0.07 \mathrm{~mm}^{-1}$

$T=120 \mathrm{~K}$

Needle, colorless

$0.70 \times 0.04 \times 0.03 \mathrm{~mm}$

12334 measured reflections

2118 independent reflections

1714 reflections with $I>2 \sigma(I)$

$R_{\text {int }}=0.059$

$\theta_{\max }=27.0^{\circ}, \theta_{\min }=1.4^{\circ}$

$h=-37 \rightarrow 37$

$k=-13 \rightarrow 14$

$l=-6 \rightarrow 6$

Secondary atom site location: difference Fourier map

Hydrogen site location: difference Fourier map

All $\mathrm{H}$-atom parameters refined

$w=1 /\left[\sigma^{2}\left(F_{\mathrm{o}}^{2}\right)+(0.03 P)^{2}+0.6 P\right]$

where $P=\left(F_{\mathrm{o}}^{2}+2 F_{\mathrm{c}}{ }^{2}\right) / 3$

$(\Delta / \sigma)_{\max }<0.001$

$\Delta \rho_{\max }=0.19 \mathrm{e} \AA^{-3}$

$\Delta \rho_{\min }=-0.18$ e $\AA^{-3}$

Special details

Geometry. All e.s.d.'s (except the e.s.d. in the dihedral angle between two 1.s. planes) are estimated using the full covariance matrix. The cell e.s.d.'s are taken into account individually in the estimation of e.s.d.'s in distances, angles and torsion angles; correlations between e.s.d.'s in cell parameters are only used when they are defined by crystal symmetry. An approximate (isotropic) treatment of cell e.s.d.'s is used for estimating e.s.d.'s involving 1.s. planes. 
Refinement. Refinement of $F^{2}$ against ALL reflections. The weighted $R$-factor $w R$ and goodness of fit $S$ are based on $F^{2}$, conventional $R$-factors $R$ are based on $F$, with $F$ set to zero for negative $F^{2}$. The threshold expression of $F^{2}>\sigma\left(F^{2}\right)$ is used only for calculating $R$-factors(gt) etc. and is not relevant to the choice of reflections for refinement. $R$-factors based on $F^{2}$ are statistically about twice as large as those based on $F$, and $R$ - factors based on ALL data will be even larger.

Fractional atomic coordinates and isotropic or equivalent isotropic displacement parameters $\left(\AA^{2}\right)$

\begin{tabular}{|c|c|c|c|c|}
\hline & $x$ & $y$ & $z$ & $U_{\text {iso }} * / U_{\text {eq }}$ \\
\hline $\mathrm{O} 1$ & $0.32558(6)$ & $0.20740(15)$ & $-0.0708(4)$ & $0.0284(4)$ \\
\hline N1 & $0.30521(6)$ & $0.10559(17)$ & $-0.1791(5)$ & $0.0261(5)$ \\
\hline N2 & $0.38448(6)$ & $0.16297(17)$ & $0.3470(5)$ & 0.0195 (4) \\
\hline $\mathrm{C} 1$ & $0.27735(8)$ & $0.1321(2)$ & $-0.3629(6)$ & $0.0265(6)$ \\
\hline $\mathrm{C} 2$ & $0.26491(8)$ & $0.2533(2)$ & $-0.4549(6)$ & $0.0298(6)$ \\
\hline C3 & $0.21338(8)$ & $0.2667(3)$ & $-0.4673(6)$ & $0.0316(6)$ \\
\hline $\mathrm{C} 4$ & $0.19196(8)$ & $0.1679(3)$ & $-0.6259(7)$ & $0.0351(7)$ \\
\hline $\mathrm{C} 5$ & $0.20409(9)$ & $0.0486(3)$ & $-0.5094(7)$ & $0.0358(7)$ \\
\hline C6 & $0.25526(9)$ & $0.0314(3)$ & $-0.5012(7)$ & $0.0324(6)$ \\
\hline $\mathrm{C} 7$ & $0.41017(8)$ & $0.0510(2)$ & $0.3611(5)$ & 0.0188 \\
\hline $\mathrm{C} 8$ & $0.43432(8)$ & $0.0337(2)$ & $0.6211(5)$ & $0.0218(5)$ \\
\hline C9 & $0.45906(8)$ & $-0.0847(2)$ & $0.6313(6)$ & $0.0236(5)$ \\
\hline $\mathrm{C} 10$ & $0.42696(9)$ & $-0.1864(2)$ & $0.5793(6)$ & $0.0243(5)$ \\
\hline $\mathrm{C} 11$ & $0.40268(9)$ & $-0.1698(2)$ & $0.3212(6)$ & $0.0255(6)$ \\
\hline $\mathrm{C} 12$ & $0.37805(8)$ & $-0.0514(2)$ & $0.3108(6)$ & $0.0235(5)$ \\
\hline C13 & $0.41173(7)$ & $0.27190(19)$ & $0.3537(5)$ & $0.0188(5)$ \\
\hline $\mathrm{C} 14$ & $0.38047(8)$ & $0.3757(2)$ & $0.4116(6)$ & $0.0226(5)$ \\
\hline $\mathrm{C} 15$ & $0.40585(8)$ & $0.4933(2)$ & $0.4109(5)$ & $0.0237(6)$ \\
\hline $\mathrm{C} 16$ & $0.43019(9)$ & $0.5120(2)$ & $0.1532(6)$ & $0.0252(6)$ \\
\hline $\mathrm{C} 17$ & $0.46156(8)$ & $0.4084(2)$ & $0.0964(6)$ & $0.0244(6)$ \\
\hline C18 & $0.43562(8)$ & $0.2919(2)$ & $0.0967(5)$ & $0.0215(5)$ \\
\hline $\mathrm{H} 1 \mathrm{O}$ & $0.3443(13)$ & $0.180(4)$ & $0.074(11)$ & $0.094(15)^{*}$ \\
\hline $\mathrm{H} 2 \mathrm{~N}$ & $0.3656(8)$ & $0.167(2)$ & $0.484(6)$ & $0.014(6)^{*}$ \\
\hline $\mathrm{H} 21$ & $0.2766(11)$ & $0.265(3)$ & $-0.644(8)$ & $0.052(10)^{*}$ \\
\hline $\mathrm{H} 22$ & $0.2792(9)$ & $0.311(2)$ & $-0.338(6)$ & $0.031(7)^{*}$ \\
\hline H31 & $0.2015(10)$ & $0.265(3)$ & $-0.276(7)$ & $0.037(8)^{*}$ \\
\hline H32 & 0.2057 (9) & $0.346(2)$ & $-0.539(6)$ & $0.032(8)^{*}$ \\
\hline H41 & $0.1600(9)$ & $0.179(2)$ & $-0.621(6)$ & $0.030(7)^{*}$ \\
\hline H42 & $0.2033(11)$ & $0.170(3)$ & $-0.814(8)$ & $0.050(10)^{*}$ \\
\hline H51 & $0.1910(9)$ & $-0.014(3)$ & $-0.609(6)$ & $0.035(8)^{*}$ \\
\hline H52 & $0.1931(10)$ & $0.043(3)$ & $-0.320(7)$ & $0.045(9)^{*}$ \\
\hline H61 & $0.2671(9)$ & $0.032(3)$ & $-0.693(7)$ & $0.036(8)^{*}$ \\
\hline H62 & $0.2613(9)$ & $-0.041(3)$ & $-0.420(7)$ & $0.041(9)^{*}$ \\
\hline H7 & $0.4340(8)$ & $0.056(2)$ & $0.217(6)$ & $0.014(6)^{*}$ \\
\hline H81 & $0.4567(8)$ & $0.097(2)$ & $0.652(6)$ & $0.026(7)^{*}$ \\
\hline H82 & $0.4121(9)$ & 0.039 (2) & $0.755(6)$ & $0.026(8) *$ \\
\hline H91 & $0.4727(9)$ & $-0.094(2)$ & $0.801(7)$ & $0.025(7)^{*}$ \\
\hline H92 & $0.4848(9)$ & $-0.085(2)$ & $0.508(6)$ & $0.026(7)^{*}$ \\
\hline H101 & $0.4425(7)$ & $-0.261(2)$ & $0.585(6)$ & $0.020(6)^{*}$ \\
\hline H102 & $0.4043(9)$ & $-0.188(2)$ & $0.721(6)$ & $0.027(7)^{*}$ \\
\hline
\end{tabular}




\begin{tabular}{lllll} 
H111 & $0.3807(8)$ & $-0.236(2)$ & $0.295(6)$ & $0.027(7)^{*}$ \\
H112 & $0.4229(10)$ & $-0.173(3)$ & $0.174(7)$ & $0.041(9)^{*}$ \\
H121 & $0.3541(8)$ & $-0.047(2)$ & $0.436(6)$ & $0.011(6)^{*}$ \\
H122 & $0.3618(8)$ & $-0.043(2)$ & $0.131(6)$ & $0.017(6)^{*}$ \\
H13 & $0.4362(7)$ & $0.272(2)$ & $0.488(5)$ & $0.013(6)^{*}$ \\
H141 & $0.3560(8)$ & $0.379(2)$ & $0.279(6)$ & $0.014(6)^{*}$ \\
H142 & $0.3664(9)$ & $0.361(2)$ & $0.574(7)$ & $0.029(8)^{*}$ \\
H151 & $0.4288(8)$ & $0.496(2)$ & $0.555(6)$ & $0.023(7)^{*}$ \\
H152 & $0.3842(8)$ & $0.562(2)$ & $0.446(6)$ & $0.025(7)^{*}$ \\
H161 & $0.4075(8)$ & $0.520(2)$ & $0.016(6)$ & $0.025(7)^{*}$ \\
H162 & $0.4466(8)$ & $0.587(2)$ & $0.161(6)$ & $0.030(7)^{*}$ \\
H171 & $0.4783(9)$ & $0.419(2)$ & $-0.064(6)$ & $0.023(7)^{*}$ \\
H172 & $0.4842(10)$ & $0.406(2)$ & $0.244(7)$ & $0.034(8)^{*}$ \\
H181 & $0.4111(8)$ & $0.292(2)$ & $-0.043(6)$ & $0.022(7)^{*}$ \\
H182 & $0.4567(8)$ & $0.228(2)$ & $0.065(6)$ & $0.021(6)^{*}$ \\
\hline
\end{tabular}

Atomic displacement parameters $\left(\AA^{2}\right)$

\begin{tabular}{lllllll}
\hline & $U^{11}$ & $U^{22}$ & $U^{33}$ & $U^{12}$ & $U^{13}$ & $U^{23}$ \\
\hline O1 & $0.0275(8)$ & $0.0263(8)$ & $0.0313(11)$ & $-0.0048(7)$ & $-0.0057(9)$ & $0.0009(8)$ \\
N1 & $0.0236(9)$ & $0.0232(10)$ & $0.0315(12)$ & $-0.0010(8)$ & $0.0008(10)$ & $-0.0015(10)$ \\
N2 & $0.0184(8)$ & $0.0180(9)$ & $0.0221(11)$ & $0.0000(8)$ & $0.0005(10)$ & $0.0014(8)$ \\
C1 & $0.0205(10)$ & $0.0295(12)$ & $0.0296(15)$ & $0.0005(10)$ & $0.0019(11)$ & $0.0012(12)$ \\
C2 & $0.0239(11)$ & $0.0301(13)$ & $0.0355(16)$ & $-0.0034(10)$ & $-0.0017(12)$ & $0.0012(13)$ \\
C3 & $0.0247(12)$ & $0.0322(14)$ & $0.0380(17)$ & $0.0040(10)$ & $-0.0018(13)$ & $-0.0003(13)$ \\
C4 & $0.0208(11)$ & $0.0431(15)$ & $0.0414(19)$ & $0.0032(12)$ & $-0.0046(14)$ & $-0.0062(13)$ \\
C5 & $0.0317(14)$ & $0.0314(14)$ & $0.044(2)$ & $-0.0061(11)$ & $-0.0032(14)$ & $-0.0082(14)$ \\
C6 & $0.0312(13)$ & $0.0285(14)$ & $0.0376(17)$ & $0.0015(11)$ & $-0.0056(13)$ & $-0.0044(13)$ \\
C7 & $0.0193(10)$ & $0.0174(10)$ & $0.0197(13)$ & $0.0011(8)$ & $0.0000(10)$ & $0.0016(10)$ \\
C8 & $0.0229(11)$ & $0.0207(11)$ & $0.0218(14)$ & $0.0009(9)$ & $-0.0031(11)$ & $-0.0013(11)$ \\
C9 & $0.0238(12)$ & $0.0203(11)$ & $0.0269(15)$ & $0.0008(9)$ & $-0.0053(12)$ & $0.0011(11)$ \\
C10 & $0.0281(12)$ & $0.0180(11)$ & $0.0269(14)$ & $0.0007(10)$ & $-0.0035(12)$ & $0.0031(11)$ \\
C11 & $0.0312(12)$ & $0.0195(11)$ & $0.0260(15)$ & $-0.0026(10)$ & $-0.0054(13)$ & $-0.0008(12)$ \\
C12 & $0.0225(11)$ & $0.0212(11)$ & $0.0267(13)$ & $-0.0020(9)$ & $-0.0069(13)$ & $0.0014(11)$ \\
C13 & $0.0190(10)$ & $0.0149(11)$ & $0.0224(13)$ & $0.0002(8)$ & $-0.0012(10)$ & $-0.0004(10)$ \\
C14 & $0.0251(11)$ & $0.0195(11)$ & $0.0232(13)$ & $0.0029(9)$ & $0.0044(11)$ & $-0.0004(11)$ \\
C15 & $0.0289(12)$ & $0.0175(11)$ & $0.0246(14)$ & $0.0005(9)$ & $0.0017(12)$ & $-0.0025(11)$ \\
C16 & $0.0287(12)$ & $0.0190(12)$ & $0.0278(14)$ & $-0.0007(10)$ & $-0.0007(12)$ & $0.0029(11)$ \\
C17 & $0.0229(12)$ & $0.0217(12)$ & $0.0286(15)$ & $-0.0039(9)$ & $0.0040(12)$ & $0.0025(11)$ \\
C18 & $0.0207(10)$ & $0.0190(11)$ & $0.0247(14)$ & $0.0001(9)$ & $0.0029(11)$ & $-0.0021(11)$ \\
& & & & & & \\
\hline
\end{tabular}

Geometric parameters $\left(\AA,{ }^{\circ}\right)$

\begin{tabular}{llll}
\hline $\mathrm{O} 1-\mathrm{N} 1$ & $1.419(3)$ & $\mathrm{C} 9-\mathrm{C} 10$ & $1.519(3)$ \\
$\mathrm{O} 1-\mathrm{H} 1 \mathrm{O}$ & $0.98(5)$ & $\mathrm{C} 9-\mathrm{H} 91$ & $0.97(3)$ \\
$\mathrm{N} 1-\mathrm{C} 1$ & $1.292(3)$ & $\mathrm{C} 9-\mathrm{H} 92$ & $0.99(3)$ \\
$\mathrm{N} 2-\mathrm{C} 13$ & $1.477(3)$ & $\mathrm{C} 10-\mathrm{C} 11$ & $1.524(4)$ \\
$\mathrm{N} 2-\mathrm{C} 7$ & $1.484(3)$ & $\mathrm{C} 10-\mathrm{H} 101$ & $0.97(3)$
\end{tabular}




\begin{tabular}{|c|c|c|c|}
\hline $\mathrm{N} 2-\mathrm{H} 2 \mathrm{~N}$ & $0.90(3)$ & $\mathrm{C} 10-\mathrm{H} 102$ & $0.99(3)$ \\
\hline $\mathrm{C} 1-\mathrm{C} 6$ & $1.499(4)$ & $\mathrm{C} 11-\mathrm{C} 12$ & $1.530(3)$ \\
\hline $\mathrm{C} 1-\mathrm{C} 2$ & $1.503(4)$ & C11-H111 & $1.00(3)$ \\
\hline $\mathrm{C} 2-\mathrm{C} 3$ & $1.534(3)$ & $\mathrm{C} 11-\mathrm{H} 112$ & $0.97(4)$ \\
\hline $\mathrm{C} 2-\mathrm{H} 21$ & $1.04(4)$ & $\mathrm{C} 12-\mathrm{H} 121$ & $0.96(3)$ \\
\hline $\mathrm{C} 2-\mathrm{H} 22$ & $0.98(3)$ & $\mathrm{C} 12-\mathrm{H} 122$ & $1.05(3)$ \\
\hline $\mathrm{C} 3-\mathrm{C} 4$ & $1.527(4)$ & $\mathrm{C} 13-\mathrm{C} 18$ & $1.520(4)$ \\
\hline $\mathrm{C} 3-\mathrm{H} 31$ & $1.05(4)$ & $\mathrm{C} 13-\mathrm{C} 14$ & $1.528(3)$ \\
\hline $\mathrm{C} 3-\mathrm{H} 32$ & $1.00(3)$ & $\mathrm{C} 13-\mathrm{H} 13$ & $1.00(3)$ \\
\hline $\mathrm{C} 4-\mathrm{C} 5$ & $1.525(5)$ & $\mathrm{C} 14-\mathrm{C} 15$ & $1.532(3)$ \\
\hline $\mathrm{C} 4-\mathrm{H} 41$ & $0.95(3)$ & $\mathrm{C} 14-\mathrm{H} 141$ & $1.00(3)$ \\
\hline $\mathrm{C} 4-\mathrm{H} 42$ & $1.03(4)$ & $\mathrm{C} 14-\mathrm{H} 142$ & $0.95(3)$ \\
\hline $\mathrm{C} 5-\mathrm{C} 6$ & $1.528(4)$ & $\mathrm{C} 15-\mathrm{C} 16$ & $1.527(4)$ \\
\hline C5-H51 & $0.96(3)$ & $\mathrm{C} 15-\mathrm{H} 151$ & $1.01(3)$ \\
\hline $\mathrm{C} 5-\mathrm{H} 52$ & $1.03(4)$ & $\mathrm{C} 15-\mathrm{H} 152$ & $1.03(3)$ \\
\hline C6-H61 & $1.05(4)$ & $\mathrm{C} 16-\mathrm{C} 17$ & $1.528(3)$ \\
\hline C6-H62 & $0.94(3)$ & $\mathrm{C} 16-\mathrm{H} 161$ & $0.98(3)$ \\
\hline $\mathrm{C} 7-\mathrm{C} 12$ & $1.525(3)$ & $\mathrm{C} 16-\mathrm{H} 162$ & $0.98(3)$ \\
\hline $\mathrm{C} 7-\mathrm{C} 8$ & $1.532(3)$ & $\mathrm{C} 17-\mathrm{C} 18$ & $1.529(3)$ \\
\hline $\mathrm{C} 7-\mathrm{H} 7$ & $1.03(3)$ & C17-H171 & $0.97(3)$ \\
\hline $\mathrm{C} 8-\mathrm{C} 9$ & $1.532(3)$ & $\mathrm{C} 17-\mathrm{H} 172$ & $1.01(4)$ \\
\hline $\mathrm{C} 8-\mathrm{H} 81$ & $0.99(3)$ & $\mathrm{C} 18-\mathrm{H} 181$ & $1.02(3)$ \\
\hline $\mathrm{C} 8-\mathrm{H} 82$ & $0.96(3)$ & $\mathrm{C} 18-\mathrm{H} 182$ & $0.97(2)$ \\
\hline $\mathrm{N} 1-\mathrm{O} 1-\mathrm{H} 1 \mathrm{O}$ & $106(2)$ & $\mathrm{H} 91-\mathrm{C} 9-\mathrm{H} 92$ & $105(2)$ \\
\hline $\mathrm{C} 1-\mathrm{N} 1-\mathrm{O} 1$ & $111.7(2)$ & $\mathrm{C} 9-\mathrm{C} 10-\mathrm{C} 11$ & $110.8(2)$ \\
\hline $\mathrm{C} 13-\mathrm{N} 2-\mathrm{C} 7$ & $115.91(16)$ & $\mathrm{C} 9-\mathrm{C} 10-\mathrm{H} 101$ & $111.4(14)$ \\
\hline $\mathrm{C} 13-\mathrm{N} 2-\mathrm{H} 2 \mathrm{~N}$ & $105.8(15)$ & $\mathrm{C} 11-\mathrm{C} 10-\mathrm{H} 101$ & $111.0(17)$ \\
\hline $\mathrm{C} 7-\mathrm{N} 2-\mathrm{H} 2 \mathrm{~N}$ & $109.1(15)$ & $\mathrm{C} 9-\mathrm{C} 10-\mathrm{H} 102$ & $107.7(16)$ \\
\hline $\mathrm{N} 1-\mathrm{C} 1-\mathrm{C} 6$ & $116.7(2)$ & $\mathrm{C} 11-\mathrm{C} 10-\mathrm{H} 102$ & $109.2(17)$ \\
\hline $\mathrm{N} 1-\mathrm{C} 1-\mathrm{C} 2$ & $127.0(2)$ & $\mathrm{H} 101-\mathrm{C} 10-\mathrm{H} 102$ & $107(2)$ \\
\hline $\mathrm{C} 6-\mathrm{C} 1-\mathrm{C} 2$ & $116.3(2)$ & $\mathrm{C} 10-\mathrm{C} 11-\mathrm{C} 12$ & $111.3(2)$ \\
\hline $\mathrm{C} 1-\mathrm{C} 2-\mathrm{C} 3$ & $110.3(2)$ & $\mathrm{C} 10-\mathrm{C} 11-\mathrm{H} 111$ & $109.5(17)$ \\
\hline $\mathrm{C} 1-\mathrm{C} 2-\mathrm{H} 21$ & $109.2(19)$ & $\mathrm{C} 12-\mathrm{C} 11-\mathrm{H} 111$ & $110.0(14)$ \\
\hline $\mathrm{C} 3-\mathrm{C} 2-\mathrm{H} 21$ & $106.1(19)$ & $\mathrm{C} 10-\mathrm{C} 11-\mathrm{H} 112$ & $113.0(19)$ \\
\hline $\mathrm{C} 1-\mathrm{C} 2-\mathrm{H} 22$ & $108.2(17)$ & $\mathrm{C} 12-\mathrm{C} 11-\mathrm{H} 112$ & $107.2(18)$ \\
\hline $\mathrm{C} 3-\mathrm{C} 2-\mathrm{H} 22$ & $112.7(17)$ & H111-C11-H112 & $106(2)$ \\
\hline $\mathrm{H} 21-\mathrm{C} 2-\mathrm{H} 22$ & $110(2)$ & $\mathrm{C} 7-\mathrm{C} 12-\mathrm{C} 11$ & $111.54(19)$ \\
\hline $\mathrm{C} 4-\mathrm{C} 3-\mathrm{C} 2$ & $111.2(2)$ & $\mathrm{C} 7-\mathrm{C} 12-\mathrm{H} 121$ & $107.8(14)$ \\
\hline $\mathrm{C} 4-\mathrm{C} 3-\mathrm{H} 31$ & $110.5(17)$ & $\mathrm{C} 11-\mathrm{C} 12-\mathrm{H} 121$ & $111.9(14)$ \\
\hline $\mathrm{C} 2-\mathrm{C} 3-\mathrm{H} 31$ & $107.0(16)$ & $\mathrm{C} 7-\mathrm{C} 12-\mathrm{H} 122$ & $111.3(13)$ \\
\hline $\mathrm{C} 4-\mathrm{C} 3-\mathrm{H} 32$ & $111.8(17)$ & $\mathrm{C} 11-\mathrm{C} 12-\mathrm{H} 122$ & $109.5(14)$ \\
\hline $\mathrm{C} 2-\mathrm{C} 3-\mathrm{H} 32$ & $109.3(15)$ & $\mathrm{H} 121-\mathrm{C} 12-\mathrm{H} 122$ & $104.6(19)$ \\
\hline $\mathrm{H} 31-\mathrm{C} 3-\mathrm{H} 32$ & $107(2)$ & $\mathrm{N} 2-\mathrm{C} 13-\mathrm{C} 18$ & $111.0(2)$ \\
\hline $\mathrm{C} 5-\mathrm{C} 4-\mathrm{C} 3$ & $110.1(3)$ & $\mathrm{N} 2-\mathrm{C} 13-\mathrm{C} 14$ & $108.67(18)$ \\
\hline $\mathrm{C} 5-\mathrm{C} 4-\mathrm{H} 41$ & $109.8(17)$ & $\mathrm{C} 18-\mathrm{C} 13-\mathrm{C} 14$ & $109.7(2)$ \\
\hline $\mathrm{C} 3-\mathrm{C} 4-\mathrm{H} 41$ & $107.5(17)$ & $\mathrm{N} 2-\mathrm{C} 13-\mathrm{H} 13$ & $114.3(14)$ \\
\hline $\mathrm{C} 5-\mathrm{C} 4-\mathrm{H} 42$ & $108.4(18)$ & $\mathrm{C} 18-\mathrm{C} 13-\mathrm{H} 13$ & $105.5(14)$ \\
\hline
\end{tabular}




\begin{tabular}{|c|c|c|c|}
\hline $\mathrm{C} 3-\mathrm{C} 4-\mathrm{H} 42$ & $110.8(18)$ & $\mathrm{C} 14-\mathrm{C} 13-\mathrm{H} 13$ & $107.6(14)$ \\
\hline $\mathrm{H} 41-\mathrm{C} 4-\mathrm{H} 42$ & $110(3)$ & $\mathrm{C} 13-\mathrm{C} 14-\mathrm{C} 15$ & $112.03(19)$ \\
\hline $\mathrm{C} 4-\mathrm{C} 5-\mathrm{C} 6$ & $111.0(2)$ & $\mathrm{C} 13-\mathrm{C} 14-\mathrm{H} 141$ & $109.4(14)$ \\
\hline $\mathrm{C} 4-\mathrm{C} 5-\mathrm{H} 51$ & $110.5(18)$ & $\mathrm{C} 15-\mathrm{C} 14-\mathrm{H} 141$ & $109.0(14)$ \\
\hline $\mathrm{C} 6-\mathrm{C} 5-\mathrm{H} 51$ & $108.7(16)$ & $\mathrm{C} 13-\mathrm{C} 14-\mathrm{H} 142$ & $107.7(17)$ \\
\hline $\mathrm{C} 4-\mathrm{C} 5-\mathrm{H} 52$ & $110.8(18)$ & $\mathrm{C} 15-\mathrm{C} 14-\mathrm{H} 142$ & $111.5(17)$ \\
\hline $\mathrm{C} 6-\mathrm{C} 5-\mathrm{H} 52$ & $106.2(18)$ & $\mathrm{H} 141-\mathrm{C} 14-\mathrm{H} 142$ & $107(2)$ \\
\hline $\mathrm{H} 51-\mathrm{C} 5-\mathrm{H} 52$ & $109(3)$ & $\mathrm{C} 16-\mathrm{C} 15-\mathrm{C} 14$ & $110.8(2)$ \\
\hline $\mathrm{C} 1-\mathrm{C} 6-\mathrm{C} 5$ & $110.4(2)$ & $\mathrm{C} 16-\mathrm{C} 15-\mathrm{H} 151$ & $108.8(16)$ \\
\hline $\mathrm{C} 1-\mathrm{C} 6-\mathrm{H} 61$ & $107.6(16)$ & $\mathrm{C} 14-\mathrm{C} 15-\mathrm{H} 151$ & $110.6(15)$ \\
\hline $\mathrm{C} 5-\mathrm{C} 6-\mathrm{H} 61$ & $107.7(16)$ & $\mathrm{C} 16-\mathrm{C} 15-\mathrm{H} 152$ & $110.1(17)$ \\
\hline $\mathrm{C} 1-\mathrm{C} 6-\mathrm{H} 62$ & $112(2)$ & $\mathrm{C} 14-\mathrm{C} 15-\mathrm{H} 152$ & $110.8(13)$ \\
\hline $\mathrm{C} 5-\mathrm{C} 6-\mathrm{H} 62$ & $108.2(17)$ & $\mathrm{H} 151-\mathrm{C} 15-\mathrm{H} 152$ & $106(2)$ \\
\hline $\mathrm{H} 61-\mathrm{C} 6-\mathrm{H} 62$ & $111(3)$ & $\mathrm{C} 15-\mathrm{C} 16-\mathrm{C} 17$ & $110.3(2)$ \\
\hline $\mathrm{N} 2-\mathrm{C} 7-\mathrm{C} 12$ & $109.04(18)$ & $\mathrm{C} 15-\mathrm{C} 16-\mathrm{H} 161$ & $108.5(16)$ \\
\hline $\mathrm{N} 2-\mathrm{C} 7-\mathrm{C} 8$ & $113.1(2)$ & $\mathrm{C} 17-\mathrm{C} 16-\mathrm{H} 161$ & $110.5(16)$ \\
\hline $\mathrm{C} 12-\mathrm{C} 7-\mathrm{C} 8$ & $110.0(2)$ & $\mathrm{C} 15-\mathrm{C} 16-\mathrm{H} 162$ & $108.7(19)$ \\
\hline $\mathrm{N} 2-\mathrm{C} 7-\mathrm{H} 7$ & $105.8(14)$ & $\mathrm{C} 17-\mathrm{C} 16-\mathrm{H} 162$ & $111.9(15)$ \\
\hline $\mathrm{C} 12-\mathrm{C} 7-\mathrm{H} 7$ & $110.2(14)$ & $\mathrm{H} 161-\mathrm{C} 16-\mathrm{H} 162$ & $107(2)$ \\
\hline $\mathrm{C} 8-\mathrm{C} 7-\mathrm{H} 7$ & $108.7(15)$ & $\mathrm{C} 16-\mathrm{C} 17-\mathrm{C} 18$ & $111.17(19)$ \\
\hline $\mathrm{C} 9-\mathrm{C} 8-\mathrm{C} 7$ & $111.4(2)$ & $\mathrm{C} 16-\mathrm{C} 17-\mathrm{H} 171$ & $112.2(16)$ \\
\hline $\mathrm{C} 9-\mathrm{C} 8-\mathrm{H} 81$ & $108.3(14)$ & $\mathrm{C} 18-\mathrm{C} 17-\mathrm{H} 171$ & $111.4(16)$ \\
\hline $\mathrm{C} 7-\mathrm{C} 8-\mathrm{H} 81$ & $111.0(17)$ & $\mathrm{C} 16-\mathrm{C} 17-\mathrm{H} 172$ & $106.0(17)$ \\
\hline $\mathrm{C} 9-\mathrm{C} 8-\mathrm{H} 82$ & $111.2(16)$ & $\mathrm{C} 18-\mathrm{C} 17-\mathrm{H} 172$ & $108.1(17)$ \\
\hline $\mathrm{C} 7-\mathrm{C} 8-\mathrm{H} 82$ & $107.7(17)$ & $\mathrm{H} 171-\mathrm{C} 17-\mathrm{H} 172$ & $108(2)$ \\
\hline $\mathrm{H} 81-\mathrm{C} 8-\mathrm{H} 82$ & $107(2)$ & $\mathrm{C} 13-\mathrm{C} 18-\mathrm{C} 17$ & $111.3(2)$ \\
\hline $\mathrm{C} 10-\mathrm{C} 9-\mathrm{C} 8$ & $111.2(2)$ & $\mathrm{C} 13-\mathrm{C} 18-\mathrm{H} 181$ & $106.6(15)$ \\
\hline $\mathrm{C} 10-\mathrm{C} 9-\mathrm{H} 91$ & $109.9(16)$ & $\mathrm{C} 17-\mathrm{C} 18-\mathrm{H} 181$ & $110.5(14)$ \\
\hline $\mathrm{C} 8-\mathrm{C} 9-\mathrm{H} 91$ & $108.8(15)$ & $\mathrm{C} 13-\mathrm{C} 18-\mathrm{H} 182$ & $109.4(16)$ \\
\hline $\mathrm{C} 10-\mathrm{C} 9-\mathrm{H} 92$ & $111.2(15)$ & $\mathrm{C} 17-\mathrm{C} 18-\mathrm{H} 182$ & $109.1(14)$ \\
\hline $\mathrm{C} 8-\mathrm{C} 9-\mathrm{H} 92$ & $110.6(15)$ & $\mathrm{H} 181-\mathrm{C} 18-\mathrm{H} 182$ & $110(2)$ \\
\hline $\mathrm{O} 1-\mathrm{N} 1-\mathrm{C} 1-\mathrm{C} 6$ & $177.2(2)$ & $\mathrm{C} 8-\mathrm{C} 9-\mathrm{C} 10-\mathrm{C} 11$ & $55.6(3)$ \\
\hline $\mathrm{O} 1-\mathrm{N} 1-\mathrm{C} 1-\mathrm{C} 2$ & $-2.2(3)$ & $\mathrm{C} 9-\mathrm{C} 10-\mathrm{C} 11-\mathrm{C} 12$ & $-55.5(3)$ \\
\hline $\mathrm{N} 1-\mathrm{C} 1-\mathrm{C} 2-\mathrm{C} 3$ & $-129.7(3)$ & $\mathrm{N} 2-\mathrm{C} 7-\mathrm{C} 12-\mathrm{C} 11$ & $179.7(2)$ \\
\hline $\mathrm{C} 6-\mathrm{C} 1-\mathrm{C} 2-\mathrm{C} 3$ & $50.8(3)$ & $\mathrm{C} 8-\mathrm{C} 7-\mathrm{C} 12-\mathrm{C} 11$ & $-55.8(3)$ \\
\hline $\mathrm{C} 1-\mathrm{C} 2-\mathrm{C} 3-\mathrm{C} 4$ & $-52.8(3)$ & $\mathrm{C} 10-\mathrm{C} 11-\mathrm{C} 12-\mathrm{C} 7$ & $56.2(3)$ \\
\hline $\mathrm{C} 2-\mathrm{C} 3-\mathrm{C} 4-\mathrm{C} 5$ & $58.0(3)$ & $\mathrm{C} 7-\mathrm{N} 2-\mathrm{C} 13-\mathrm{C} 18$ & $74.5(3)$ \\
\hline $\mathrm{C} 3-\mathrm{C} 4-\mathrm{C} 5-\mathrm{C} 6$ & $-58.7(3)$ & $\mathrm{C} 7-\mathrm{N} 2-\mathrm{C} 13-\mathrm{C} 14$ & $-164.8(2)$ \\
\hline $\mathrm{N} 1-\mathrm{C} 1-\mathrm{C} 6-\mathrm{C} 5$ & $129.0(3)$ & $\mathrm{N} 2-\mathrm{C} 13-\mathrm{C} 14-\mathrm{C} 15$ & $-177.6(2)$ \\
\hline $\mathrm{C} 2-\mathrm{C} 1-\mathrm{C} 6-\mathrm{C} 5$ & $-51.5(3)$ & $\mathrm{C} 18-\mathrm{C} 13-\mathrm{C} 14-\mathrm{C} 15$ & $-56.1(3)$ \\
\hline $\mathrm{C} 4-\mathrm{C} 5-\mathrm{C} 6-\mathrm{C} 1$ & $54.2(4)$ & $\mathrm{C} 13-\mathrm{C} 14-\mathrm{C} 15-\mathrm{C} 16$ & $56.3(3)$ \\
\hline $\mathrm{C} 13-\mathrm{N} 2-\mathrm{C} 7-\mathrm{C} 12$ & $-171.5(2)$ & $\mathrm{C} 14-\mathrm{C} 15-\mathrm{C} 16-\mathrm{C} 17$ & $-55.6(3)$ \\
\hline $\mathrm{C} 13-\mathrm{N} 2-\mathrm{C} 7-\mathrm{C} 8$ & $65.8(3)$ & $\mathrm{C} 15-\mathrm{C} 16-\mathrm{C} 17-\mathrm{C} 18$ & $56.4(3)$ \\
\hline $\mathrm{N} 2-\mathrm{C} 7-\mathrm{C} 8-\mathrm{C} 9$ & $178.02(19)$ & $\mathrm{N} 2-\mathrm{C} 13-\mathrm{C} 18-\mathrm{C} 17$ & $176.48(18)$ \\
\hline $\mathrm{C} 12-\mathrm{C} 7-\mathrm{C} 8-\mathrm{C} 9$ & $55.9(3)$ & $\mathrm{C} 14-\mathrm{C} 13-\mathrm{C} 18-\mathrm{C} 17$ & $56.4(3)$ \\
\hline $\mathrm{C} 7-\mathrm{C} 8-\mathrm{C} 9-\mathrm{C} 10$ & $-56.3(3)$ & $\mathrm{C} 16-\mathrm{C} 17-\mathrm{C} 18-\mathrm{C} 13$ & $-57.5(3)$ \\
\hline
\end{tabular}


Hydrogen-bond geometry $\left(A,{ }^{\circ}\right)$

\begin{tabular}{lllll}
\hline$D-\mathrm{H} \cdots A$ & $D-\mathrm{H}$ & $\mathrm{H} \cdots A$ & $D \cdots A$ & $D-\mathrm{H} \cdots A$ \\
\hline $\mathrm{O} 1-\mathrm{H} 1 O \cdots \mathrm{N} 2$ & $0.98(5)$ & $1.85(5)$ & $2.818(3)$ & $167(4)$ \\
$\mathrm{N} 2-\mathrm{H} 2 N \cdots \mathrm{O} 1^{\mathrm{i}}$ & $0.90(3)$ & $2.62(3)$ & $3.509(3)$ & $167(2)$ \\
$\mathrm{N} 2-\mathrm{H} 2 N \cdots \mathrm{N} 1^{\mathrm{i}}$ & $0.90(3)$ & $2.59(3)$ & $3.451(3)$ & $160(2)$ \\
$\mathrm{C} 2-\mathrm{H} 21 \cdots \mathrm{O} 1^{\mathrm{ii}}$ & $1.04(3)$ & $2.71(3)$ & $3.686(3)$ & $155(2)$
\end{tabular}

Symmetry codes: (i) $x, y, z+1$; (ii) $x, y, z-1$. 